\title{
Les installations de l'IRSN dédiées à la métrologie des neutrons
}

\author{
V. GRESSIER ${ }^{1}$, J.L. POCHAT ${ }^{1}$
}

(Manuscrit reçu le 4 août 2005, accepté le 4 octobre 2005)

RÉSUMÉ La responsabilité des références françaises pour la fluence et les grandeurs dosimétriques neutroniques est du ressort du laboratoire de métrologie et de dosimétrie des neutrons de l'Institut de radioprotection et de sûreté nucléaire (IRSN). C'est dans ce cadre, que l'institut développe et exploite, à Cadarache, des installations produisant des champs neutroniques de référence pour des utilisations diverses. Certaines (sources radioactives émettrice neutrons respectant la norme ISO 8529-1) sont utilisées pour effectuer des étalonnages de routine de dispositifs de mesure. D'autres installations produisent des champs neutroniques « réalistes » simulant les champs rencontrés sur les postes de travail en modérant les champs neutroniques générés à partir de sources radioactives ou d'accélérateurs. Enfin, l'installation AMANDE dont l'inauguration a eu lieu en mai 2005 produit des champs de neutrons monoénergétiques d'énergie comprise entre $2 \mathrm{keV}$ et $20 \mathrm{MeV}$ à partir de l'interaction de particules chargées accélérées jusqu'à $4 \mathrm{MeV}$ sur différentes cibles. Avec l'arrivée de cette dernière installation, l'IRSN offre un plateau technique très complet pour la production de tous les types de champ neutronique recommandés par les normes internationales pour l'étalonnage des instruments de détection des neutrons.

ABSTRACT The IRSN installations dedicated to the metrology of neutrons.

The Institute for Radiation Protection and Nuclear Safety (IRSN) is in charge of the French references for neutron fluence and dosimetric quantities. Within this context the institute develops and exploits, at Cadarache, facilities producing reference neutron fields for different purposes. Some (radioactive sources emitting neutrons respecting ISO 8529-1 standard) are used for routine calibrations of measuring instruments. Others facilities produce "realistic" neutron fields, simulating fields encountered at workplaces by moderating neutron fields created by radioactive sources or accelerators. Finally, AMANDE facility, inaugurated in may 2005, produces mono-energetic neutron fields in the energy range from $2 \mathrm{keV}$ to $20 \mathrm{MeV}$ by nuclear reaction within different targets of charged particles accelerated up to $4 \mathrm{MeV}$. With this new facility, IRSN offers a very complete facility set to produce any type of neutron field recommended by international standards for calibration of neutron detection systems.

Keywords: neutron fields / source / accelerators / calibration

1 IRSN, DRPH/SDE/Laboratoire de métrologie et dosimétrie neutrons, bâtiment 159, B.P. 3, 13115 Saint Paul Lez Durance, France. 


\section{Introduction}

Différents secteurs d'activités génèrent des neutrons nécessitant la mise en place d'une surveillance dosimétrique associée : l'industrie nucléaire au travers des centrales, des installations de production et de retraitement du combustible ou des centres de recherches associés, mais également le secteur militaire, les laboratoires de recherche et le domaine médical (dans l'environnement des accélérateurs) ainsi que le spatial. Le nombre de personnes ainsi surveillées est de l'ordre de 20000 en France et de 60000 en Europe (Ambrosi et al., 2000).

La nouvelle directive européenne Euratom 96/29 et ses déclinaisons dans les textes de lois nationaux conduisent à un renforcement de la dosimétrie. De plus, différentes études ont montré les difficultés d'assurer de manière satisfaisante la surveillance dosimétrique des travailleurs exposés aux neutrons (Bordy et al., 2000). Ainsi, l'amélioration de la dosimétrie des neutrons utilisés pour assurer la radioprotection des travailleurs constitue un enjeu important pour les années à venir, tant en France qu'au plan international.

\section{La chaîne de métrologie neutron en France}

En France, le Laboratoire national de métrologie et d'essais (LNE) a pour mission de préparer et de mettre en œuvre la politique nationale de métrologie. Dans le domaine des rayonnements ionisants, le Laboratoire national Henri Becquerel (LNHB), au CEA est le laboratoire responsable de la métrologie fondamentale ainsi que des références primaires et de transfert. Ce laboratoire n'ayant pas d'activité en dosimétrie des neutrons, la responsabilité pour les grandeurs dosimétriques neutroniques a été transférée à l'IRSN, laboratoire associé au LNE.

Sur le site de Cadarache, l'IRSN développe et exploite des moyens de transfert et d'étalonnage nécessaires aux raccordements des laboratoires accrédités. À ce jour, ce rôle est tenu par les sources neutroniques radioactives de l'irradiateur VAN GOGH, décrit ci-dessous, accréditées par le Comité français d'accréditation (COFRAC) pour des prestations d'étalonnage. À moyen terme, toutes les installations de l'IRSN seront intégrées au sein des possibilités de mesures et d'étalonnage accréditées du laboratoire associé.

\section{Champs neutroniques de référence}

Pour répondre au besoin d'amélioration de la dosimétrie des neutrons, l'IRSN développe et exploite des installations produisant des champs neutroniques de 
référence. Ces champs pour les étalonnages des instruments de radioprotection sont définis dans les normes de l'Organisation internationale de normalisation (ISO, 2000, 2001) auxquelles se réfèrent également les normes de la commission électrotechnique internationale (CEI, 1994, 2003, 2005) relatifs aux instruments eux-mêmes.

Ces rayonnements de référence peuvent être scindés en plusieurs catégories selon leur utilisation.

\section{1. Étalonnage de routine}

Pour l'étalonnage de routine de dispositifs de mesure des neutrons ayant déjà été soumis à l'ensemble des tests de fonctionnement sous rayonnement lors de leur conception ou homologation, les sources radioactives ${ }^{241} \mathrm{AmBe}$ (neutrons produits par réaction $(\alpha, \mathrm{n})$ ) et ${ }^{252} \mathrm{Cf}$ (neutrons issus de la fission spontanée du radionucléide) sont recommandées par la norme ISO 8529-1 car l'énergie moyenne des neutrons (4,2 MeV pour $\mathrm{AmBe}$ et $2,1 \mathrm{MeV}$ pour ${ }^{252} \mathrm{Cf}$ ) est du même ordre que celle des neutrons produits par réaction de fission dans le cycle du combustible.

Les sources radioactives sont les sources de neutrons les plus répandues du fait de la relative facilité à déterminer la fluence (nombre de neutrons par $\mathrm{cm}^{2}$ ) et les grandeurs dosimétriques à partir de la mesure de l'activité initiale de la source lors de sa création, et du coût modéré de l'exploitation de telles sources de neutrons.

Toutefois ces sources ne peuvent raisonnablement être utilisées pour étudier de nouveaux appareils de détection des neutrons ou dosimètres. En effet, l'étalonnage réalisé auprès de ces sources radioactives ne sera strictement valable que pour des champs neutroniques dont la distribution en énergie est similaire à celle des sources $\mathrm{AmBe}$ ou ${ }^{252} \mathrm{Cf}$, ce qui est assez rarement le cas sur les postes de travail.

\section{2. Étalonnage dans des champs "réalistes"}

Les dosimètres personnels et, en général, tous les appareils de mesure des neutrons ont une réponse dépendant de l'énergie des neutrons. Il peut donc s'avérer impossible d'étalonner correctement un instrument qui sera utilisé dans un champ neutronique dont les distributions en énergie et angulaire diffèrent significativement du champ de rayonnement de référence utilisé pour l'étalonnage.

C'est à cet effet que la norme ISO 12789-1 a introduit la notion de « champ neutronique simulant ceux de postes de travail», également appelé «champ 
neutronique réaliste ». Ces champs neutroniques, une fois précisément caractérisés, peuvent être utilisés comme champ de référence et sont recommandés dans les nouvelles normes de la CEI.

L'objectif est de pouvoir étalonner tout appareil dans un champ neutronique le plus proche possible de celui dans lequel il sera utilisé par la suite. L'étalonnage dans de tels champs réalistes permet d'obtenir une dosimétrie plus fiable et plus adaptée à chaque poste de travail.

\subsection{Détermination de la réponse d'appareils de mesure en fonction de l'énergie des neutrons}

L'étude de la variation de la réponse des instruments de mesure avec l'énergie des neutrons permet de valider les réponses neutroniques calculées des instruments et/ou de déterminer et de vérifier l'adéquation des moyens d'étalonnage de ces instruments. Pour réaliser une telle étude, les champs neutroniques utilisés doivent avoir une distribution en énergie limitée sur une plage étroite en énergie.

Les champs neutroniques recommandés pour une telle étude sont :

- les champs de neutrons thermiques, où l'énergie des neutrons est inférieure à $0,5 \mathrm{eV}$, produits par réacteurs ;

- les champs de neutrons monoénergétiques ou quasi-monoénergétiques produits par faisceau filtré de réacteur $(2 \mathrm{keV}, 24 \mathrm{keV}$ et $144 \mathrm{keV})$ ou par réactions nucléaires à l'aide d'accélérateurs de particules chargées. Les réactions nucléaires utilisées sont ${ }^{45} \mathrm{Sc}(\mathrm{p}, \mathrm{n})$ pour les énergies $2 \mathrm{keV}$ et $24 \mathrm{keV}$ recommandées par la norme ISO, ${ }^{7} \mathrm{Li}(\mathrm{p}, \mathrm{n})$ pour les énergies $144 \mathrm{keV}, 250 \mathrm{keV}$ et $565 \mathrm{keV}, \mathrm{T}(\mathrm{p}, \mathrm{n})$ pour les énergies $1,2 \mathrm{MeV}$ et 2,5 MeV, D(d, n) pour les énergies $2,8 \mathrm{MeV}$ et $5 \mathrm{MeV}$ et enfin $\mathrm{T}(\mathrm{d}, \mathrm{n})$ pour les énergies de $14,8 \mathrm{MeV}$ et $19 \mathrm{MeV}$.

\subsection{Installations de l'IRSN}

Pour chaque méthode d'étalonnage décrite précédemment, l'IRSN dispose des installations correspondantes :

- étalonnage de routine : sources radioactives neutroniques ${ }^{252} \mathrm{Cf}$ et ${ }^{241} \mathrm{AmBe}$ de l'irradiateur VAN GOGH ;

- champ neutronique réaliste : source de ${ }^{252} \mathrm{Cf}$ modérée, installation SIGMA et dispositif CANEL ;

- étude de la réponse en fonction de l'énergie des neutrons :

O champ thermique: à défaut de réacteur, l'IRSN utilise l'installation SIGMA et une correction par mesure avec écran de cadmium ;

○ champs monoénergétiques : accélérateurs J25, T400 et AMANDE. 
Contrairement à la plupart des installations équivalentes dans le monde, les installations de l'IRSN produisent les champs de rayonnement neutronique dans des halls expérimentaux dont les parois sont construites en matériau léger. En outre, les points de production des neutrons (sources, cibles, etc.) sont situés à plusieurs mètres de hauteur par rapport au sol. Cela permet, dans les deux cas de limiter les neutrons diffusés qui sont une source de bruit de fond compliquant notablement la détermination précise des champs de rayonnement.

\section{Description du plateau technique de l'IRSN}

\subsection{Irradiateur VAN GOGH}

L'irradiateur VAN GOGH (Van Ryckeghem et al., 2002) est constitué des deux principales sources radioactives recommandées par la norme ISO 8529-1: ${ }^{241} \mathrm{AmBe}$ et ${ }^{252} \mathrm{Cf}$.

Lorsqu'elles ne sont pas utilisées, ces sources sont entreposées dans un conteneur en polyéthylène placé au pied de l'irradiateur. Grâce à de l'air comprimé dans un tube-guide, les sources sont amenées à leur position d'irradiation située à 3,2 m au-dessus du sol, ce qui permet de limiter le bruit de fond dû aux neutrons diffusés par le sol. Un banc d'étalonnage motorisé permet de placer les instruments au niveau de la source et à toute distance entre 0 et $2 \mathrm{~m}$, la distance d'irradiation conventionnelle étant $75 \mathrm{~cm}$.

Au-delà de l'activité d'étalonnage de routine, cet irradiateur a pour mission de raccorder les laboratoires accrédités aux références nationales pour la dosimétrie des neutrons. Dans ce cadre, les activités d'étalonnage à l'aide de cet irradiateur sont accréditées par le COFRAC.

\section{- Sources «nues »}

La source de ${ }^{241} \mathrm{Am}-\mathrm{Be}$ est recouverte d'un capuchon de plomb de $1 \mathrm{~mm}$ d'épaisseur afin de limiter le rayonnement photonique émis par la source ; la ${ }^{252} \mathrm{Cf}$ est utilisée sans aucun écran.

La distribution en énergie de la fluence neutronique associée à chacune de ces sources, dans la norme ISO 8529-1, est représentée sur la figure 1.

\section{- Source ${ }^{252}$ Cf modérée}

Afin de produire des champs « réalistes », et selon les recommandations de la norme ISO $8529-1$, la source de ${ }^{252} \mathrm{Cf}$ est placée au centre d'une sphère 


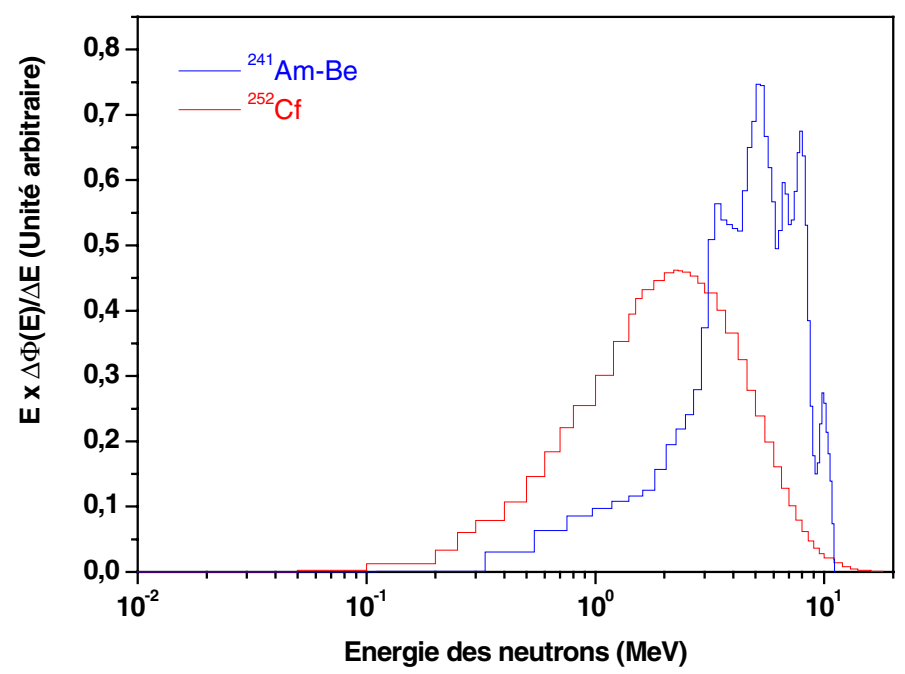

Figure 1 - Distributions en énergie de la fluence neutronique des sources de ${ }^{241} \mathrm{AmBe}$ et ${ }^{252} \mathrm{Cf} *$. Neutron fluence energy distributions of ${ }^{241} \mathrm{AmBe}$ and ${ }^{252} \mathrm{Cf}$ sources.

modératrice d'eau lourde de $15 \mathrm{~cm}$ de rayon avec une coquille d'aluminium de 1,2 mm d'épaisseur, elle-même recouverte d'une coquille de $0,8 \mathrm{~mm}$ de cadmium. La configuration sans cadmium n'est pas recommandée par l'ISO mais représente également un champ réaliste, avec une c9omposante thermique.

Les deux distributions en énergie de la fluence obtenues par modération sont représentées sur la figure 2 et peuvent être utilisées pour s'approcher un peu plus de la distribution en énergie de la fluence neutronique mesurée sur un poste de travail tel que, par exemple, un crayon de combustible MOX situé derrière un mur en béton (Lacoste et al., 2004b).

- Débits de fluence et d'équivalent de dose

Les débits de fluence et débits d'équivalent de dose (individuel et ambiant) à $75 \mathrm{~cm}$ des sources sont présentés dans le tableau I. Ces valeurs sont directement déterminées à partir de la mesure de l'activité neutronique de la source réalisée dans un bain de manganèse par le National Physical Laboratory (NPL), laboratoire national de métrologie de Grande Bretagne) pour la source d'AmBe et par le LNHB pour la source de ${ }^{252} \mathrm{Cf}$.

* Une version en couleurs des figures est disponible sur www.edpsciences.org/radiopro/ 

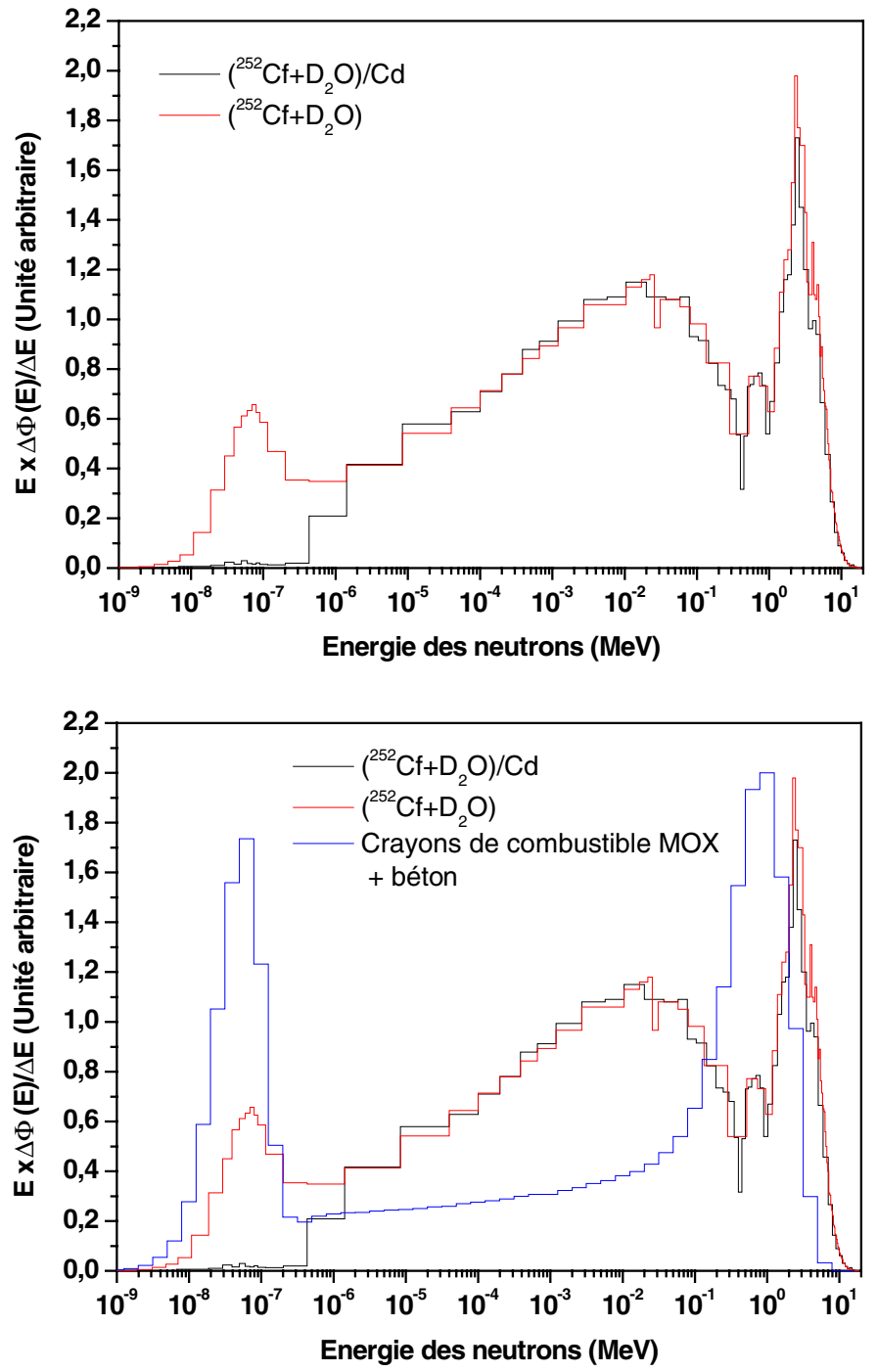

Figure 2 - Distributions en énergie de la fluence neutronique issues de la source de ${ }^{252}$ Cf modérée et comparaison avec la distribution mesurée à un poste de travail (crayon de combustible MOX situés derrière un mur en béton).

Neutron fluence energy distribution of moderated ${ }^{252} \mathrm{Cf}$ source and comparison with measured energy distribution at workplace (MOX fuel rod located behind a concrete wall). 
TABLEAU I

Fluence et équivalent de dose (individuel et ambiant) à $75 \mathrm{~cm}$ de la source, intégrant les neutrons diffusés par l'environnement, à la date du $1^{\mathrm{er}}$ mai 2005.

Fluence an dose equivalent, with scattering neutrons contribution included, at $75 \mathrm{~cm}$ from the source the 1st of May 2005.

\begin{tabular}{ccccc} 
& ${ }^{\mathbf{2 4 1}} \mathbf{A m}-\mathbf{B e}$ & ${ }^{\mathbf{2 5 2}} \mathbf{C f}$ & $\left({ }^{\mathbf{2 5 2}} \mathbf{C f}+\mathbf{D}_{\mathbf{2}} \mathbf{O}\right) / \mathbf{C d}$ & ${ }^{\mathbf{2 5 2}} \mathbf{C f}+\mathbf{D}_{\mathbf{2}} \mathbf{O}$ \\
\hline Émission $\left(\mathrm{s}^{-1}\right)$ & $4 \times 10^{7}$ & $3 \times 10^{8}$ & $3 \times 10^{8}$ & $3 \times 10^{8}$ \\
$\dot{\Phi}_{\mathrm{n}}\left(\mathrm{cm}^{-2} \mathrm{~s}^{-1}\right)$ & $542 \pm 9$ & 4500 & 3900 & 4500 \\
$\dot{\mathrm{H}}^{*}(10)\left(\mu \mathrm{Sv} \mathrm{h}^{-1}\right)$ & $769 \pm 33$ & 6100 & 1480 & 1520 \\
$\dot{\mathrm{H}}_{\mathrm{p}}\left(10,0^{\circ}\right)\left(\mu \mathrm{Sv} \mathrm{h}^{-1}\right)$ & $806 \pm 35$ & 6300 & 1550 & 1590 \\
\hline
\end{tabular}

\subsection{Installation SIGMA}

L'installation SIGMA produit des champs neutroniques réalistes à forte composante thermique. Elle est constituée d'un empilement cubique de graphite de $150 \mathrm{~cm}$ d'arête contenant 6 sources radioactives d ${ }^{241} \mathrm{AmBe}$ (dont l'émission neutronique totale est $(1,91 \pm 0,04) \times 10^{8} \mathrm{~s}^{-1}$ au $\left.01 / 09 / 03\right)$, le graphite servant de modérateur. Les appareils à irradier sont placés à une distance de $50 \mathrm{~cm}$ du bloc de graphite. Un banc d'étalonnage motorisé devrait être installé fin 2005.

La représentation graphique de la distribution en énergie des «neutrons » à $50 \mathrm{~cm}$ de SIGMA est présentée sur la figure 3. Cette distribution a été déterminée par simulation MCNP (Briesmeister, 2000) et confirmée expérimentalement pour les énergies au-delà de $100 \mathrm{keV}$ par des mesures à l'aide de détecteurs à protons de recul, dans le domaine thermique par une mesure d'activation de feuille d'or et sur tout le domaine en énergie par des mesures avec des sphères de Bonner (Lacoste et al., 2004a).

Les débits de fluence et d'équivalent de dose (individuel et ambiant) ainsi que la contribution pour différents domaines d'énergie sont présentés dans le tableau II. La fluence neutronique est composée à $90 \%$ de neutrons «thermiques » (énergie inférieure à $0,5 \mathrm{eV}$ ), mais la contribution des neutrons thermiques aux équivalents de dose n'est que de l'ordre de $40 \%$. Le champ neutronique produit par cette installation est multidirectionnel (les angles d'incidence des neutrons au point de calibrage sont compris entre 0 et $50^{\circ}, 30^{\circ}$ étant l'angle privilégié), ce qui explique les différences notables entre équivalents de dose ambiant et personnel (Lacoste et Gressier, 2005).

L'installation SIGMA peut être utilisée en tant que :

- champ de neutrons réaliste : par exemple, ce champ est représentatif de certains postes de travail mesurés à proximité de réacteur thermique à eau pressurisé (Lacoste et Muller, 2004) ainsi que le montre la figure 4 ; 


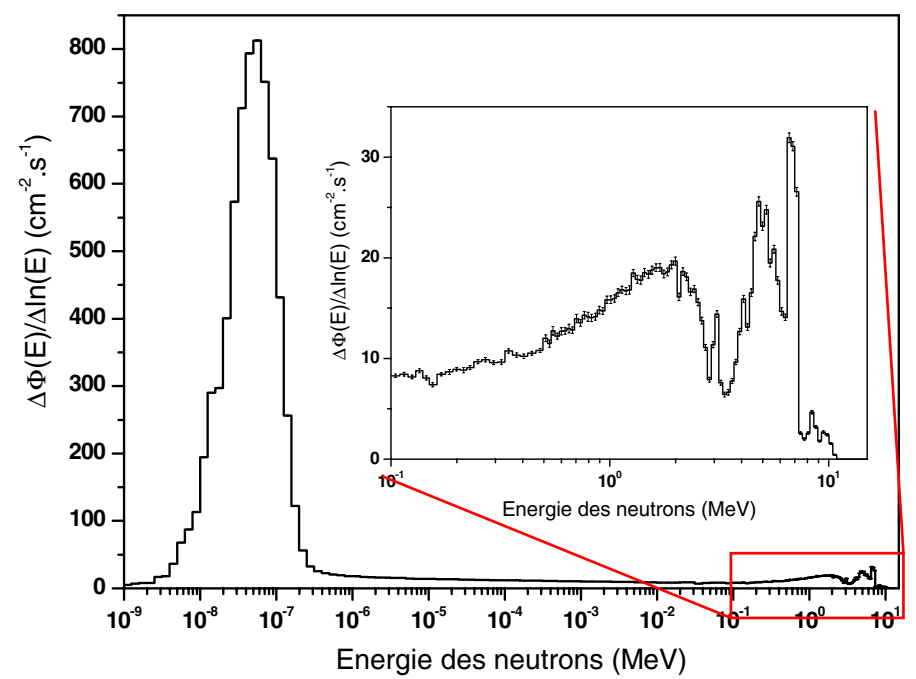

Figure 3 -Distribution en énergie de la fluence neutronique à $50 \mathrm{~cm}$ de SIGMA avec détails sur les neutrons d'énergie supérieure à $100 \mathrm{keV}$.

Neutron fluence energy distribution of neutron fluence at $50 \mathrm{~cm}$ from SIGMA.

TABLEAU II

Débits de fluence et d'équivalent de dose à la position usuelle de calibrage de SIGMA.

Fluence rate and dose equivalent at the usual calibration position of SIGMA.

\begin{tabular}{ccccc}
\hline $\mathbf{E}_{\mathbf{n}}(\mathbf{M e V})$ & Total & $\mathbf{1 0}^{-11} \leq \mathbf{E}_{\mathbf{n}}<\mathbf{5} \times \mathbf{1 0}^{-\mathbf{7}}$ & $\mathbf{5} \times \mathbf{1 0}^{-7} \leq \mathbf{E}_{\mathbf{n}}<\mathbf{1 0}^{-\mathbf{1}}$ & $\mathbf{1 0}^{-\mathbf{1}} \leq \mathbf{E}_{\mathbf{n}}$ \\
\hline$\dot{\Phi}_{\mathrm{n}}\left(\mathrm{cm}^{-2} \mathrm{~s}^{-1}\right)$ & $1767 \pm 55$ & $88,4 \%$ & $8,3 \%$ & $3,3 \%$ \\
$\dot{\mathrm{H}}^{*}(10)\left(\mu \mathrm{Sv} \mathrm{h}^{-1}\right)$ & $144 \pm 5$ & $43,9 \%$ & $4,8 \%$ & $51,3 \%$ \\
$\dot{\mathrm{H}}_{\mathrm{p}}\left(10,0^{\circ}\right)\left(\mu \mathrm{Sv} \mathrm{h}^{-1}\right)$ & $132 \pm 5$ & $35,8 \%$ & $2,9 \%$ & $61,3 \%$ \\
\hline
\end{tabular}

- champ de neutrons thermiques : pour cela une mesure supplémentaire doit être effectuée avec un écran de cadmium entourant l'instrument de mesure afin d'obtenir la contribution à la lecture de l'appareil des neutrons supérieurs à $0,5 \mathrm{eV}$, qui sera soustraite à la lecture obtenue sans écran. De cette manière, seuls les neutrons du premier groupe en énergie du tableau II sont mesurés.

\subsection{Les accélérateurs J25 et T400}

Les générateurs de neutrons J25 et T400 sont deux accélérateurs amenant des deutons (noyau du deutérium) à des énergies maximales de $150 \mathrm{keV}$ et $400 \mathrm{keV}$. 


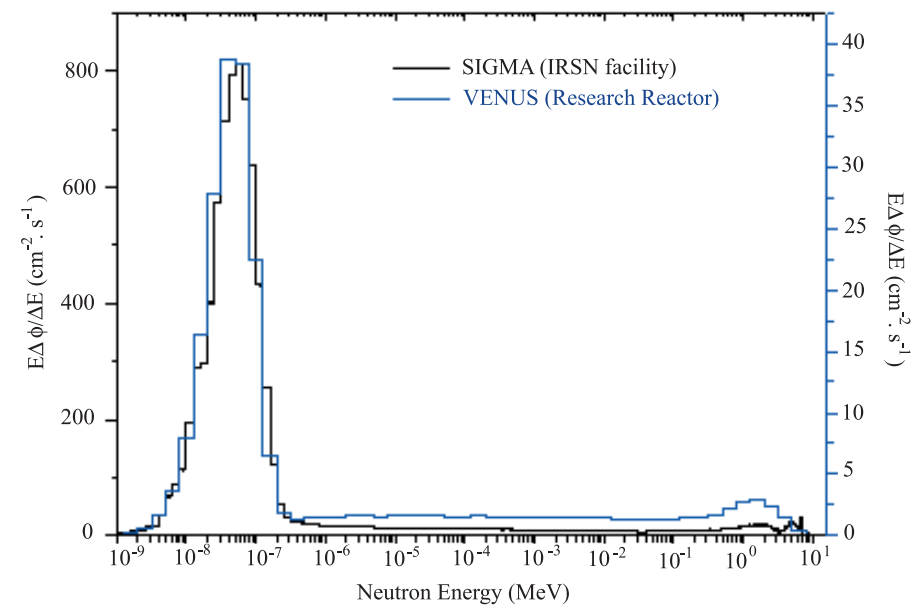

Figure 4 -Distribution en énergie des «neutrons »à SIGMA compare à celle mesurée à un poste de travail dans le réacteur de recherché VENUS.

Neutron energy distribution at SIGMA compared to measured distribution at a workplace in the VENUS reactor.

Ces deutons accélérés sont envoyés sur des cibles de titane deutérié (T400) ou titane tritié (J25) où leur interaction avec les atomes de deutérium ou de tritium va générer des champs de neutrons quasi-monoénergétiques respectivement de l'ordre de $3 \mathrm{MeV}$ et $15 \mathrm{MeV}$ ainsi que le montre le tableau III.

TABLEAU III

Réaction nucléaire sur les accélérateurs T400 et $\mathbf{J} 25$.

Nuclear reaction at $\mathrm{T} 400$ and $\mathrm{J} 25$ accelerators.

\begin{tabular}{cccccc}
\hline Accélérateur & $\begin{array}{c}\text { Faisceau de } \\
\text { particules }\end{array}$ & Cibles & Réactions & $\begin{array}{c}\text { Énergie des particules } \\
\text { incidentes }\end{array}$ & $\begin{array}{c}\text { Énergie moyenne } \\
\left(0^{\circ}\right)\end{array}$ \\
\hline T400 & deutons & deutérium (TiD) & ${ }^{2} \mathrm{H}(\mathrm{d}, \mathrm{n}){ }^{3} \mathrm{He}$ & 150 à $400 \mathrm{keV}$ & 2,9 à $3,2 \mathrm{MeV}$ \\
$\mathrm{J} 25$ & deutons & tritium (TiT) & ${ }^{3} \mathrm{H}(\mathrm{d}, \mathrm{n}){ }^{4} \mathrm{He}$ & 50 à $150 \mathrm{keV}$ & 14,4 à $14,7 \mathrm{MeV}$ \\
\hline
\end{tabular}

La fonction principale de ces générateurs est de fournir des hauts flux de neutrons pour des applications en radioprotection. Le fonctionnement nominal du T400 est à une énergie de $350 \mathrm{keV}$ et du J25 à une énergie de $120 \mathrm{keV}$.

Les cibles utilisées sont des cibles épaisses arrêtant complètement le faisceau de particules chargées et le faisceau de deutons est pollué par environ $20 \%$ d'ions diatomiques. Il en résulte une distribution en énergie des neutrons constituée de 

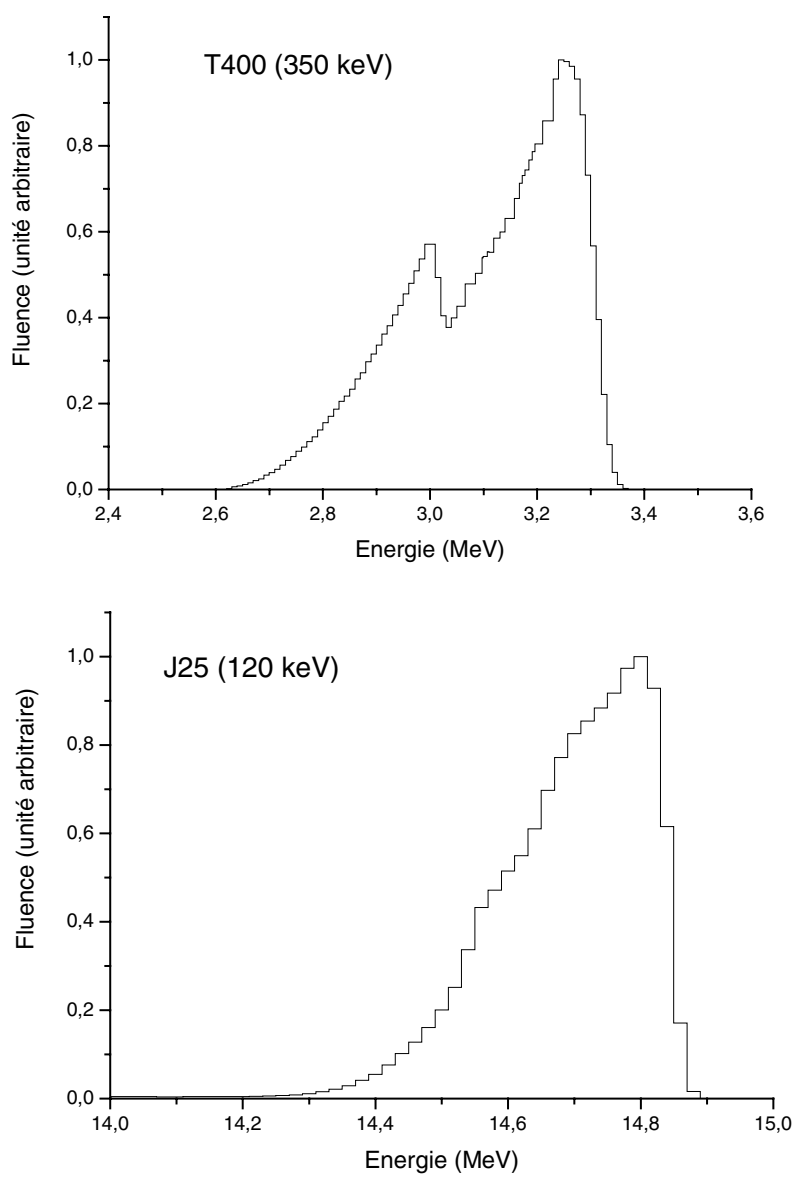

Figure 5 - Distribution en énergie de la fluence neutronique produite par les accélérateurs T400 (réaction $\left.\mathrm{D}(d, n)^{3} \mathrm{He}\right)$ et $\mathrm{J} 25$ (réaction $\mathrm{T}(d, n)^{4} \mathrm{He}$ ) en fonctionnement nominal avec $20 \%$ d'ions diatomiques).

Neutron fluence energy distribution produced by $\mathrm{T} 400$ accelerator $\left(\mathrm{D}(\mathrm{d}, \mathrm{n})^{3} \mathrm{He}\right)$ reaction) and J25 accelerator $\left(\mathrm{T}(\mathrm{d}, \mathrm{n})^{4} \mathrm{He}\right)$ reaction) in nominal run with $20 \%$ diatomic ions.

deux pics quasi-monoénergétiques de plusieurs centaines de keV de largeur à mihauteur se superposant comme le montre la figure 5 (Gressier et al., 2002).

La fluence neutronique est mesurée en utilisant la méthode de la «particule associée $»: 3$ diodes silicium sont placées à l'arrière de la cible (angle de $175^{\circ}$ ) et détectent les particules alpha (J25) ou les protons (T400) émis lors de la production des neutrons. 
Les appareils à irradier peuvent être placés entre 0 et $2 \mathrm{~m}$ de la cible dans l'axe du faisceau de deutons $\left(0^{\circ}\right)$.

Les neutrons diffusés représentent $14 \%$ de la fluence à $1 \mathrm{~m}$ de la cible dans le cas du T400. Les caractéristiques des champs neutroniques et les valeurs maximales des débits de fluence et d'équivalent de dose personnel sont données dans le tableau IV. La résolution en énergie neutron $\Delta \mathrm{E}_{\mathrm{n}}$ correspond à la largeur à mi-hauteur du pic «mono-énergétique ». Les valeurs maximales des débits de fluence et d'équivalent de dose personnel sont données pour une distance de $1 \mathrm{~m}$ par rapport à la cible, dans l'axe du faisceau de deutons et correspondent à 3000 coups/s et 2400 coups/s respectivement dans les diodes du T400 et du J25.

TABLEAU IV

Caractéristiques des champs neutroniques produits par les accélérateurs J25 et T400 en fonctionnement nominal.

Specifications of neutron fields produced by $\mathrm{J} 25$ and $\mathrm{T} 400$ in nominal run.

\begin{tabular}{|c|c|c|c|c|c|c|}
\hline Accélérateur & $\begin{array}{l}\text { Énergie des } \\
\text { deutons }\end{array}$ & $\begin{array}{l}\mathbf{E}_{\mathbf{n}}\left(0^{\circ}\right) \\
(\mathrm{MeV})\end{array}$ & $\Delta \mathbf{E}_{\mathbf{n}} / \mathbf{E}_{\mathbf{n}}$ & $\begin{array}{c}\text { Emission } \\
\text { neutronique }\left(\mathrm{s}^{-1}\right)\end{array}$ & $\underset{\left(10^{4} \mathrm{~cm}^{-2} \cdot \mathrm{s}^{-1}\right)}{\mathrm{d} / \mathrm{dt} \max }$ & $\begin{array}{c}\mathrm{dHp}\left(10,0^{\circ}\right) / \mathrm{dt} \max \\
(\mathrm{mSv} / \mathrm{h})\end{array}$ \\
\hline $\mathrm{T} 400$ & $350 \mathrm{keV}$ & 3,1 & $10 \%$ & $2,2 \times 10^{9}$ & $3,6 \pm 0,2$ & $56 \pm 3$ \\
\hline $\mathrm{J} 25$ & $120 \mathrm{keV}$ & 14,7 & $1,7 \%$ & $1,4 \times 10^{10}$ & $11,8 \pm 0,7$ & $240 \pm 13$ \\
\hline
\end{tabular}

Les accélérateurs $\mathrm{J} 25$ et T400 sont utilisés pour toute irradiation à haut débit avec des faisceaux quasi-monoénergétiques à 3,1 ou $14,7 \mathrm{MeV}$ où une très bonne résolution en énergie du pic monoénergétique n'est pas requise. Ils complètent ainsi les possibilités offertes par l'accélérateur AMANDE dont les débits de fluence et d'équivalents de dose neutronique sont plus faibles, comme détaillé dans le tableau VII.

\subsection{Dispositif CANEL}

«CANEL » (CAnon à NEutron Lent) est un dispositif modulaire qui vient se placer autour de la cible des générateurs de neutrons J25 et T400 pour produire des champs neutroniques « réalistes ». C'est un dispositif unique au monde cité à titre d'exemple dans la norme ISO 12789.

Le principe de CANEL est de générer un spectre de neutrons de fission et de le modérer à l'aide d'éléments représentatifs des dispositifs de modération des neutrons mis en place autour des réacteurs de l'industrie nucléaire. Les «neutrons » quasi-monoénergétiques de 3,1 et 14,7 MeV, issus du T400 et du J25 respectivement, rencontrent tout d'abord une coquille d' uranium appauvri où ils sont convertis 


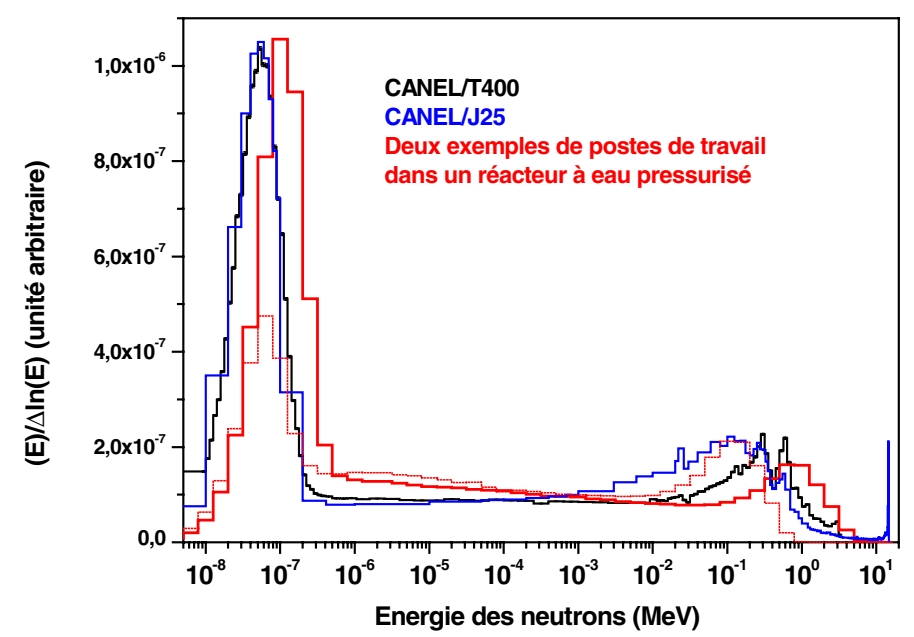

Figure 6-Distribution en énergie de la fluence neutronique obtenue à l'aide de dispositif "CANEL" utilisé avec l'accélérateur T400 ou J25 et comparaison avec des distributions en énergie mesurées à des postes de travail dans un réacteur eau pressurisé.

Neutron fluence energy distribution at the calibration position in front "CANEL" system used using T400 or J25 accelerator and comparison with energy distribution measured at PWR workplaces.

par réaction de fission. Ces neutrons de fission traversent ensuite un écran de fer puis une lentille d'eau, le tout étant placé dans un conduit en polyéthylène de $70 \mathrm{~cm}$ de diamètre et de $1 \mathrm{~m}$ de longueur. Ce conduit en polyéthylène permet de confiner les neutrons diffusés à l'intérieur de l'enceinte et de diriger le champ neutronique vers l'avant. La taille des écrans de fer et d'eau (en cours de remplacement par du polyéthylène) peut être variée et est différente dans le cas de l'utilisation de CANEL sur le J25 (configuration CANEL/J25) ou le T400 (CANEL/T400).

Le point d'étalonnage des appareils est situé dans l'axe du faisceau de deutons à $30 \mathrm{~cm}$ de la sortie de CANEL dans le cas CANEL/J25 et $50 \mathrm{~cm}$ dans le cas CANEL/T400.

Les distributions en énergie de la fluence neutronique à ces positions ont été déterminées par simulation avec le code MCNP (Chartier et al., 1992 ; Lacoste et Gressier, 2004) et validées expérimentalement par des campagnes internationales de spectrométrie utilisant des détecteurs à protons de recul et des systèmes à sphères de Bonner (Thomas et al., 1997 ; Gressier et al., 2004b).

Ces distributions en énergie sont représentatives de celles des champs neutroniques rencontrés à certains postes de travail de l'industrie nucléaire (Lacoste, 2005) ainsi que le montre la figure 6. 
Les débits de fluence et d'équivalent de dose (individuel et ambiant) maxima déterminés pour différents domaines d'énergie et au point de test des détecteurs devant « CANEL », sont présentés dans le tableau V.

\section{TABLEAU V}

Débits de fluence et d'équivalent de dose ambiant maxima à $50 \mathrm{~cm}$ de CANEL/T400 et $30 \mathrm{~cm}$ de CANEL/J25.

Maxima fluence rate and ambient dose equivalent at $50 \mathrm{~cm}$ from CANEL/T400 and $30 \mathrm{~cm}$ of CANEL/J25.

\begin{tabular}{cccccc}
\hline & $\mathbf{E}_{\mathbf{n}}(\mathbf{M e V})$ & Total & $\mathbf{1 0}^{-\mathbf{1 1}} \leq \mathbf{E}_{\mathbf{n}}<\mathbf{5} \times \mathbf{1 0}^{-\mathbf{7}}$ & $\mathbf{5} \times \mathbf{1 0}^{-\mathbf{7}} \leq \mathbf{E}_{\mathbf{n}}<\mathbf{1 0}^{-\mathbf{1}}$ & $\mathbf{1 0}^{\mathbf{- 1}} \leq \mathbf{E}_{\mathbf{n}}$ \\
\hline \multirow{2}{*}{ CANEL/T400 } & $\dot{\Phi}_{\mathrm{n}}\left(\mathrm{cm}^{-2} \mathrm{~s}^{-1}\right)$ & $8150 \pm 350$ & $56,0 \%$ & $25,2 \%$ & $18,8 \%$ \\
& $\dot{\mathrm{H}} *(10)\left(\mathrm{mSv} \mathrm{h}^{-1}\right)$ & $1,32 \pm 0,07$ & $15,5 \%$ & $4,9 \%$ & $79,6 \%$ \\
& $\dot{\Phi}_{\mathrm{n}}\left(\mathrm{cm}^{-2} \mathrm{~s}^{-1}\right)$ & $262000 \pm 9000$ & $54,7 \%$ & $31,0 \%$ & $14,3 \%$ \\
CANEL/J25 & $\dot{\mathrm{H}} *(10)\left(\mathrm{mSv} \mathrm{h}^{-1}\right)$ & $31,7 \pm 4,1$ & $16,5 \%$ & $10,2 \%$ & $73,3 \%$ \\
\hline
\end{tabular}

Il n'existe à ce jour que deux configurations caractérisées pour CANEL, mais la modularité de CANEL permet de générer des spectres neutroniques de différentes formes en jouant sur le degrés de modération des neutrons et ainsi s'approcher le plus possible de la forme de la distribution en énergie des neutrons rencontrés sur un poste de travail donné.

\subsection{Installation AMANDE}

L'installation AMANDE a pour but de produire des champs de neutrons monoénergétiques. Ces champs sont obtenus à partir de particules chargées (protons et deutons) accélérées puis dirigées sur une cible constituée d'un dépôt de scandium, lithium, tritium, ou deutérium sur un mince support métallique. L'interaction des particules chargées avec les noyaux de ces éléments génère des neutrons dans tout l'espace. Les réactions nucléaires correspondantes ont la particularité de produire des neutrons d'une seule énergie pour une énergie donnée de particule chargée incidente et un angle d'émission donné des neutrons (Marion et Fowler, 1960).

AMANDE permettra également de produire des rayonnements gamma autour de $7 \mathrm{MeV}$ d'énergie par la réaction ${ }^{19} \mathrm{~F}(\mathrm{p}, \alpha \gamma){ }^{16} \mathrm{O}$ (ISO, 1996). Cette source de rayonnement complétera les moyens d'irradiation par photons de l'IRSN dont l'énergie maximale est actuellement de $1,32 \mathrm{MeV}$ (source de ${ }^{60} \mathrm{Co}$ ). L'installation sera progressivement ouverte, fin 2005 pour les besoins du service de dosimétrie de l'IRSN et courant 2006 pour les utilisateurs externes. 


\subsubsection{Accélérateur}

AMANDE est un tandetron de $2 \mathrm{MV}$, c'est-à-dire un accélérateur de type Cockroft Walton de géométrie tandem, permettant de couvrir le plus grand domaine possible en énergie de neutrons monoénergétiques avec un accélérateur de taille raisonnable. AMANDE permet d'accélérer en continu ou en mode pulsé des protons et des deutons à des énergies comprises entre $100 \mathrm{keV}$ et $4 \mathrm{MeV}$ avec une excellente résolution et une très grande stabilité en énergie $(<500 \mathrm{eV})$.

L'accélérateur AMANDE, mesure 4,5 m de longueur, Le parcours des particules chargées entre la source d'ions et la cible où sont produits les neutrons est de $35 \mathrm{~m}$ avec notamment 4 aimants de déviation : un aimant de $15^{\circ}$ après la source pour sélectionner le type d'ion à accélérer, un aimant d'analyse de $90^{\circ}$ à la sortie de l'accélérateur permettant de sélectionner précisément l'énergie désirée des particules, un aimant de $40^{\circ}$ dont le rôle est de compenser les effets de distorsion engendré par l'aimant de $90^{\circ}$ en mode pulsé et un aimant de distribution à 5 angles, un seul des angles $\left(10^{\circ}\right)$ étant utilisé à ce jour pour la ligne de faisceau d'AMANDE (Gressier et al., 2003).

\subsubsection{Domaine en énergie des neutrons}

Les réactions et les domaines en énergie correspondants des neutrons émis à $0^{\circ}$ sont donnés dans le tableau VI.

\section{TABLEAU VI}

Réactions nucléaires utilisées pour la production de neutrons monoénergétiques avec l'accélérateur AMANDE.

Nuclear reactions used to produce monoenergetic neutrons at AMANDE Accelerator.

\begin{tabular}{ccccc}
\hline $\begin{array}{c}\text { Faisceau de } \\
\text { particules }\end{array}$ & Cibles & Réactions & $\begin{array}{c}\text { Énergie particules } \\
\text { chargées }\end{array}$ & $\begin{array}{c}\text { Énergie neutrons } \\
\text { monoénergétiques }\left(0^{\circ}\right)\end{array}$ \\
\hline protons & scandium & ${ }^{45} \mathrm{Sc}(\mathrm{p}, \mathrm{n}){ }^{45} \mathrm{Ti}$ & 2,91 à $2,95 \mathrm{MeV}$ & 5,6 à $52 \mathrm{keV}$ \\
protons & lithium & ${ }^{7} \mathrm{Li}(\mathrm{p}, \mathrm{n}){ }^{7} \mathrm{Be}$ & 1,92 à $2,38 \mathrm{MeV}$ & 120 à $650 \mathrm{keV}$ \\
protons & tritium $(\mathrm{TiT})$ & ${ }^{3} \mathrm{H}(\mathrm{p}, \mathrm{n})^{3} \mathrm{He}$ & 1,15 à $4 \mathrm{MeV}$ & $290 \mathrm{keV}$ à $3,2 \mathrm{MeV}$ \\
deutons & deutérium $(\mathrm{TiD})$ & ${ }^{2} \mathrm{H}(\mathrm{d}, \mathrm{n})^{3} \mathrm{He}$ & 0,1 à $4 \mathrm{MeV}$ & 2,8 à $7,3 \mathrm{MeV}$ \\
deutons & tritium $(\mathrm{TiT})$ & ${ }^{3} \mathrm{H}(\mathrm{d}, \mathrm{n}){ }^{4} \mathrm{He}$ & 0,1 à $3,7 \mathrm{MeV}$ & 14,7 à $20,5 \mathrm{MeV}$ \\
\hline
\end{tabular}

Pour chaque réaction, il est possible d'obtenir des neutrons monoénergétiques d'énergie inférieure en se plaçant à un angle d'émission des neutrons supérieur à $0^{\circ}$. Le domaine en énergie couvert par AMANDE est ainsi de $2 \mathrm{keV}$ à $7,3 \mathrm{MeV}$ et de $12 \mathrm{MeV}$ à $20,5 \mathrm{MeV}$. 
V. GRESSIER, J.L. POCHAT

\subsubsection{Caractéristiques des champs monoénergétiques}

AMANDE a pour but de générer des faisceaux monoénergétiques de haute résolution en énergie. L'accélérateur a été conçu de manière à avoir une très faible dispersion en énergie du faisceau de particules chargées et une très grande stabilité en énergie assurée par l'aimant d'analyse à $90^{\circ}$, toutes deux étant inférieure à $500 \mathrm{eV}$. La résolution (largeur à mi-hauteur) du pic mono-énergétique dépend donc principalement de l'épaisseur de la cible et un compromis entre résolution en énergie et émission neutronique doit être fait, expliquant l'utilisation de plusieurs épaisseurs de cible. Les caractéristiques des cibles et des champs neutroniques sont résumées dans le tableau VII. Dans ce tableau, les 5 premières colonnes précisent les réactions nucléaires utilisées (avec éventuellement le type de cible), l'épaisseur du dépôt de la cible, l'énergie du faisceau de protons ou de deutons et l'énergie des neutrons à $0^{\circ}$ (sauf astérisques) suivie de la résolution en énergie du pic monoénergétique. Les colonnes suivantes donnent respectivement l'émission neutronique maximale dans tout l'espace, les débits de fluence neutronique et d'équivalent de dose personnel à $1 \mathrm{~m}$ de la cible avec pour chacun d'eux la proportion de neutrons diffusés à ajouter et enfin le courant maximal du faisceau de particules chargées considéré pour le calcul des valeurs précédentes. Quelques exemples de distributions en énergie de la fluence neutronique sont représentés sur la figure 7 .

\subsubsection{Aire expérimentale}

Un soin tout particulier a été apporté pour limiter le nombre de neutrons diffusés à un faible pourcentage des neutrons directs (non diffusés) (Gressier et al., 2004a), ainsi que le montre le tableau VII. À cet effet, la cible est située à 7,2 m du sol, au centre d'une fosse de $6 \mathrm{~m}$ de rayon entourée d'un caillebotis métallique et comme pour les autres installations, le hall expérimental de $400 \mathrm{~m}^{2}$ est entouré de parois métalliques.

Un système automatisé (bras mobiles) permet le positionnement précis (l'incertitude induite sur la fluence neutronique est inférieure à $0,1 \%$ ) et reproductible des détecteurs à une distance comprise entre $50 \mathrm{~cm}$ et 6 mètres de la cible et dans une plage angulaire comprise entre $-160^{\circ}$ et $160^{\circ}$ par rapport à l'axe du faisceau de particules chargées (voir Fig. 8). De plus, un système de régulation de température et d'hygrométrie par rideau d'air autour de la zone d'étalonnage a été implanté dans ce hall d'expérimentation afin d'assurer au point de mesure les spécifications de conditions normales de température et d'hygrométrie spécifiée dans les normes internationales de la CEI. 
TABLEAU VII

Caractéristiques des champs neutroniques monoénergétiques produits par AMANDE aux énergies recommandées par la norme ISO 8529-1.

Specifications of the monoenergetic neutron fields produced by AMANDE at the energies recommended by ISO 8529-1 standard.

\begin{tabular}{|c|c|c|c|c|c|c|c|c|c|c|}
\hline $\begin{array}{l}\text { Réaction } \\
\text { nucléaire }\end{array}$ & $\begin{array}{c}\text { Épaisseur } \\
\text { du dépôt } \\
\left(\mu \mathrm{g} / \mathrm{cm}^{2}\right)\end{array}$ & $\begin{array}{l}\mathbf{E}_{p}-\mathbf{E}_{\mathbf{d}} \\
(\mathrm{MeV})\end{array}$ & $\begin{array}{l}\operatorname{En}\left(0^{\circ}\right) \\
(\mathrm{MeV})\end{array}$ & $\Delta E_{n} / E_{n}$ & $\begin{array}{c}\text { Émission } \\
\text { neutronique } \\
\max \left(\mathrm{s}^{-1}\right)\end{array}$ & $\begin{array}{c}\mathrm{d} \Phi_{\max } / \mathrm{dt} \\
\left(0^{\circ}\right) \\
\left(\mathrm{cm}^{-2} \mathrm{~s}^{-1}\right)\end{array}$ & $\begin{array}{l}\text { neutrons } \\
\text { diffusés }\end{array}$ & $\begin{array}{c}\mathrm{dHp}_{\max } \\
\left(\mathbf{1 0 , 0 ^ { \circ } ) / \mathrm { dt }}\right. \\
\left(\mathrm{mSv} \mathrm{h}^{-1}\right)\end{array}$ & $\begin{array}{c}\text { neutrons } \\
\text { diffusés }\end{array}$ & $\begin{array}{l}\mathbf{I}_{\max } \\
(\boldsymbol{\mu} \mathbf{A})\end{array}$ \\
\hline \multirow[t]{2}{*}{${ }^{45} \mathrm{Sc}(\mathrm{p}, \mathrm{n}){ }^{45} \mathrm{Ti}$} & $\begin{array}{c}5 \\
25\end{array}$ & 2,91 & $0,002 *$ & $\begin{array}{l}30 \% \\
80 \%\end{array}$ & $\begin{array}{l}1,3 \times 10^{5} \\
6,4 \times 10^{5}\end{array}$ & $\begin{array}{l}2 \\
8\end{array}$ & $18 \%$ & $\begin{array}{l}5,2 \times 10^{-5} \\
2,6 \times 10^{-4}\end{array}$ & $19 \%$ & \multirow{2}{*}{50} \\
\hline & & 2,92 & 0,024 & $\begin{array}{l}2,5 \% \\
10 \%\end{array}$ & $\begin{array}{l}2,1 \times 10^{5} \\
1,1 \times 10^{6}\end{array}$ & $\begin{array}{c}3 \\
14\end{array}$ & $13 \%$ & $\begin{array}{l}2,0 \times 10^{-4} \\
1,0 \times 10^{-3}\end{array}$ & $9 \%$ & \\
\hline \multirow{4}{*}{$\begin{array}{l}{ }^{7} \mathrm{Li}(\mathrm{p}, \mathrm{n}){ }^{7} \mathrm{Be} \\
\text { (cible LiF) }\end{array}$} & \multirow{4}{*}{$\begin{array}{c}10 \\
40 \\
120\end{array}$} & 1,9 & $0,024 *$ & $\begin{array}{l}15 \% \\
24 \% \\
55 \%\end{array}$ & $\begin{array}{l}1,9 \times 10^{6} \\
8,0 \times 10^{6} \\
2,4 \times 10^{7}\end{array}$ & $\begin{array}{c}15 \\
65 \\
230\end{array}$ & - & $\begin{array}{c}1,1 \times 10^{-3} \\
4,7 \times 10^{-3} \\
0,02\end{array}$ & - & \multirow{3}{*}{7} \\
\hline & & 1,9 & 0,144 & $\begin{array}{c}3 \% \\
6 \% \\
14 \%\end{array}$ & $\begin{array}{l}2,1 \times 10^{6} \\
9,0 \times 10^{6} \\
2,6 \times 10^{7}\end{array}$ & $\begin{array}{c}55 \\
220 \\
670\end{array}$ & $6 \%$ & $\begin{array}{l}0,02 \\
0,11 \\
0,32\end{array}$ & $3 \%$ & \\
\hline & & 2,0 & 0,250 & $\begin{array}{l}1 \% \\
3 \% \\
6 \%\end{array}$ & $\begin{array}{l}3,0 \times 10^{6} \\
1,2 \times 10^{7} \\
3,0 \times 10^{7}\end{array}$ & $\begin{array}{c}35 \\
150 \\
350\end{array}$ & $11 \%$ & $\begin{array}{l}0,03 \\
0,11 \\
0,29\end{array}$ & $5 \%$ & \\
\hline & & 2,3 & 0,565 & $\begin{array}{c}0,5 \% \\
1 \% \\
2 \%\end{array}$ & $\begin{array}{l}3,5 \times 10^{6} \\
1,4 \times 10^{7} \\
4,0 \times 10^{7}\end{array}$ & $\begin{array}{c}100 \\
420 \\
1200\end{array}$ & $4 \%$ & $\begin{array}{r}0,1 \\
0,5 \\
1,5\end{array}$ & $3 \%$ & 6 \\
\hline \multirow{3}{*}{$\begin{array}{c}{ }^{3} \mathrm{H}(\mathrm{p}, \mathrm{n})^{3} \mathrm{He} \\
\text { (cible TiT) }\end{array}$} & \multirow{3}{*}{$\begin{array}{c}200 \\
800 \\
2000\end{array}$} & 2,0 & 1,2 & $\begin{array}{c}1,5 \% \\
5 \% \\
13 \%\end{array}$ & $\begin{array}{l}5,2 \times 10^{7} \\
2,1 \times 10^{8} \\
5,9 \times 10^{8}\end{array}$ & $\begin{array}{c}770 \\
3100 \\
8700\end{array}$ & $7 \%$ & $\begin{array}{c}1,2 \\
4,8 \\
13,6\end{array}$ & $5 \%$ & 7 \\
\hline & & 3,3 & 2,5 & $\begin{array}{l}0,6 \% \\
1,8 \% \\
4,3 \%\end{array}$ & $\begin{array}{l}5,1 \times 10^{7} \\
1,8 \times 10^{8} \\
5,3 \times 10^{8}\end{array}$ & $\begin{array}{c}1050 \\
3800 \\
10900\end{array}$ & $4 \%$ & $\begin{array}{c}1,7 \\
6,0 \\
17,1\end{array}$ & $3 \%$ & 5 \\
\hline & & 3,6 & 2,8 & $\begin{array}{l}0,5 \% \\
1,5 \% \\
3,5 \%\end{array}$ & $\begin{array}{l}3,3 \times 10^{7} \\
1,4 \times 10^{8} \\
3,9 \times 10^{8}\end{array}$ & $\begin{array}{c}680 \\
2800 \\
8000\end{array}$ & - & $\begin{array}{c}1,1 \\
4,4 \\
12,5\end{array}$ & - & 4 \\
\hline \multirow[t]{2}{*}{$\begin{array}{c}{ }^{2} \mathrm{H}(\mathrm{d}, \mathrm{n})^{3} \mathrm{He} \\
\text { (cible TiD) }\end{array}$} & $\begin{array}{c}200 \mathrm{ou} \\
800\end{array}$ & 0,1 & 2,8 & $4 \%$ & $2,1 \times 10^{6}$ & 60 & - & 0,09 & - & 30 \\
\hline & $\begin{array}{l}200 \\
800\end{array}$ & 1,8 & 5 & $\begin{array}{c}0,6 \% \\
2 \%\end{array}$ & $\begin{array}{l}1,5 \times 10^{7} \\
6,1 \times 10^{7}\end{array}$ & $\begin{array}{c}560 \\
2320\end{array}$ & $2 \%$ & $\begin{array}{c}0,85 \\
3,5\end{array}$ & $2 \%$ & 8 \\
\hline \multirow[t]{2}{*}{$\begin{array}{c}{ }^{3} \mathrm{H}(\mathrm{d}, \mathrm{n}){ }^{4} \mathrm{He} \\
\text { (cible TiT) }\end{array}$} & $\begin{array}{c}200 \\
800 \\
2000\end{array}$ & 0,2 & 14,8 & $\begin{array}{l}1,6 \% \\
(200) \\
2,5 \%\end{array}$ & $\begin{array}{l}3,9 \times 10^{8} \\
3,9 \times 10^{8}\end{array}$ & $\begin{array}{l}3300 \\
3300\end{array}$ & $7 \%$ & $\begin{array}{l}6,5 \\
6,5\end{array}$ & $5 \%$ & $\begin{array}{c}5 \\
3,5\end{array}$ \\
\hline & & 2,6 & 19 & $\begin{array}{c}0,15 \% \\
0,5 \% \\
1,4 \%\end{array}$ & $\begin{array}{l}1,2 \times 10^{7} \\
4,3 \times 10^{7} \\
1,1 \times 10^{8}\end{array}$ & $\begin{array}{c}180 \\
660 \\
1750\end{array}$ & $4 \%$ & $\begin{array}{c}0,36 \\
1,3 \\
3,4\end{array}$ & $3 \%$ & 6 \\
\hline
\end{tabular}

\subsubsection{Précision et méthode de détermination des références}

L'énergie et la fluence des champs monoénergétiques d'AMANDE sont déterminées par la méthode du temps de vol et tout un ensemble de détecteurs. 

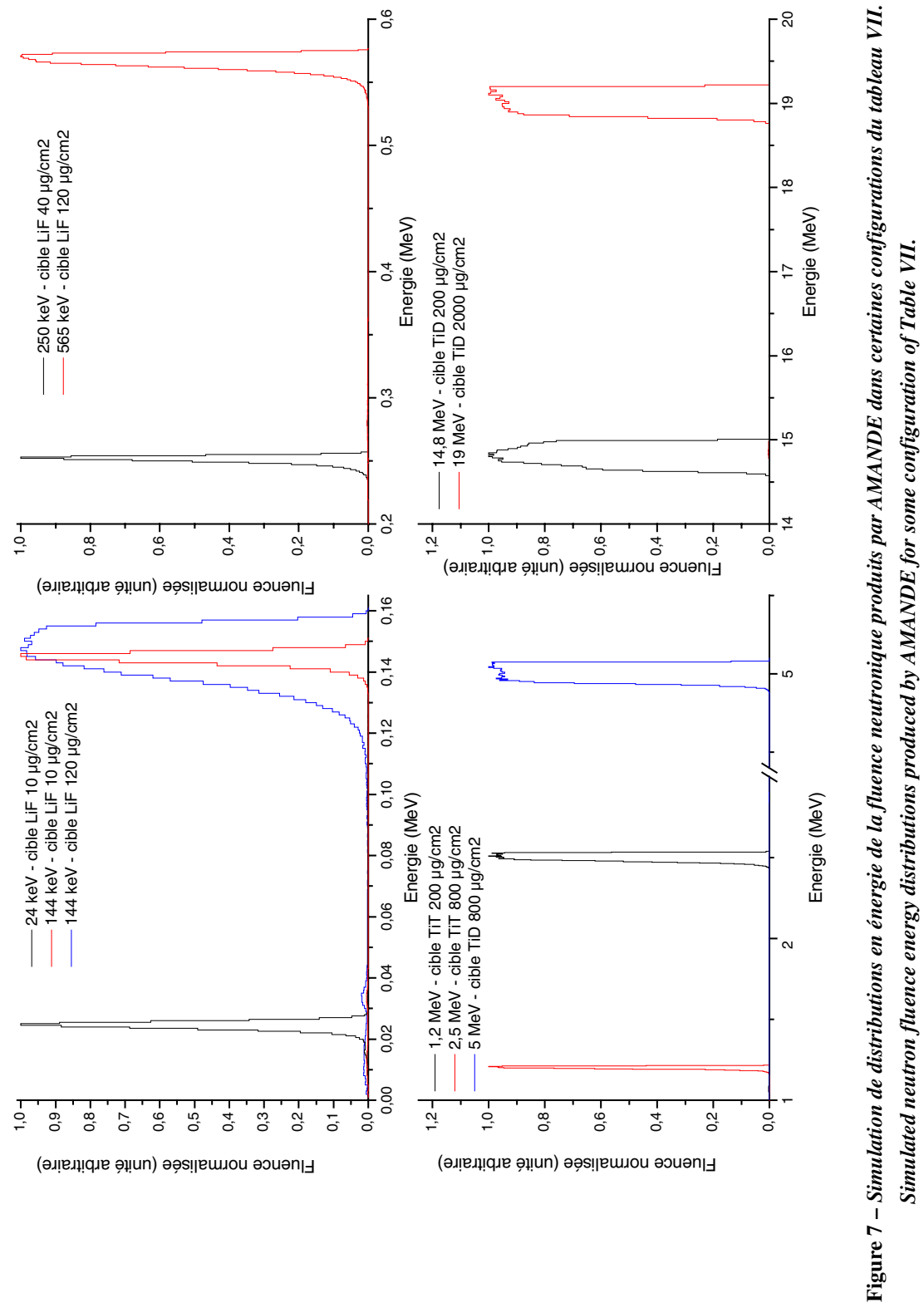


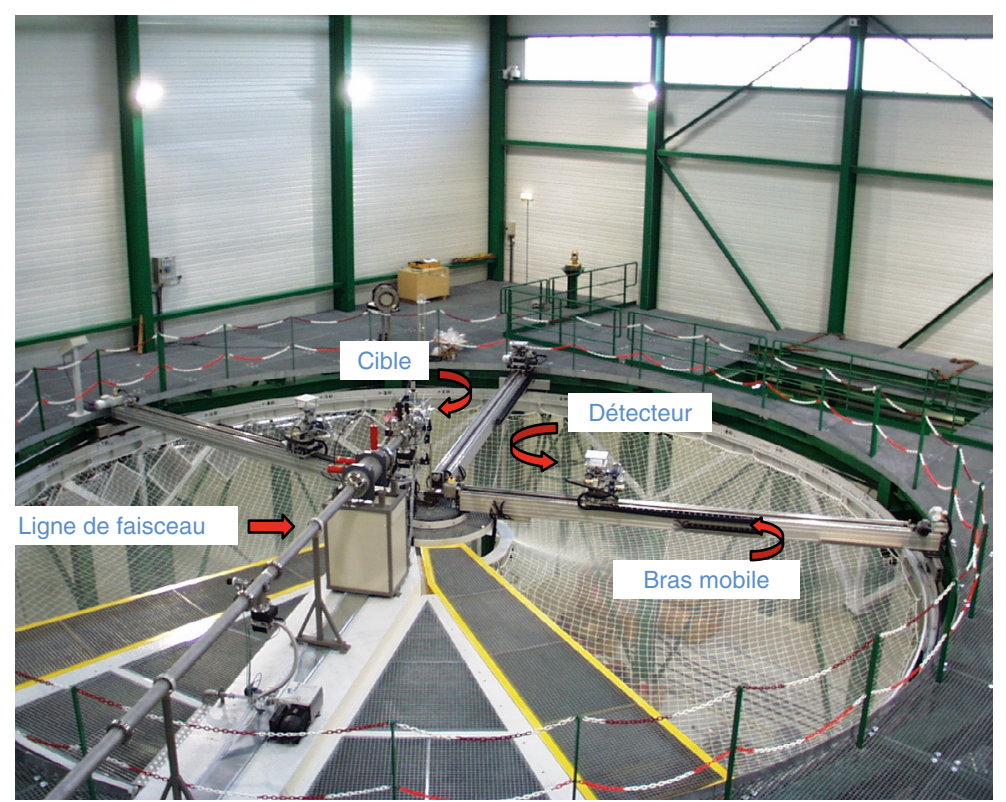

Figure 8 - Hall d'expérimentation d'AMANDE.

AMANDE experimental hall.

\section{- Références en énergie}

Les références en énergie sont données par des mesures de temps de vol, c'est-àdire la mesure du temps mis par les neutrons pour parcourir une distance donnée, ce temps étant directement lié à l'énergie des neutrons. La distance de mesure peut être comprise entre $50 \mathrm{~cm}$ et 10 mètres à $0^{\circ}$. Pour effectuer ce type de mesure, l'accélérateur fonctionne en mode pulsé avec une fréquence variable entre $62,5 \mathrm{kHz}$ et $2 \mathrm{MHz}$ et une largeur d'impulsion pouvant descendre à $0,8 \mathrm{~ns}$. L'incertitude sur l'énergie due à cette méthode est représentée en fonction de l'énergie des neutrons et de la distance sur la figure 9.

Il apparait que la détermination de l'énergie des neutrons peut être déterminée, sur toute la gamme en énergie, à moins de $1 \%$ près.

\section{- Références en fluence}

La fluence est déterminée par des détecteurs à protons de recul (Pichenot et al., 2002 ; Schmidt et al., 2002) et un long compteur (un détecteur de neutrons 


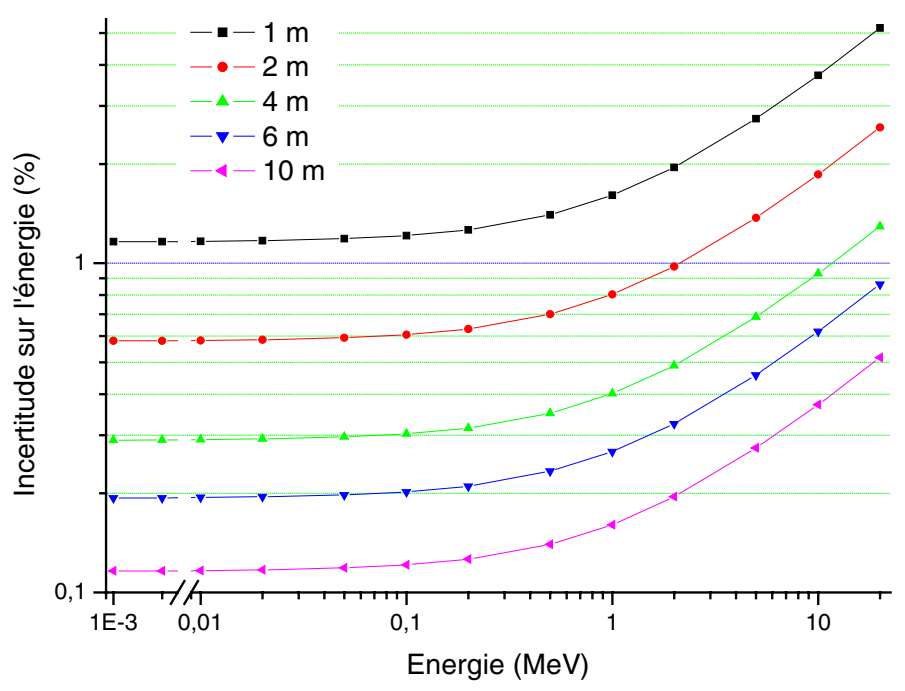

Figure 9 - Incertitude sur l'énergie des neutrons déterminée par la méthode du temps de vol à plusieurs distances entre 1 et $10 \mathrm{~m}$ pour une largeur d'impulsion de 0,8 ns et un détecteur de $2 \mathrm{~cm}$ d'épaisseur.

Energy resolution of the time of flight method at several distances between 1 and 10 meters for a 0.8 ns pulse width and a $2 \mathrm{~cm}$ thick detector.

thermiques cylindrique entouré d'une enceinte modératrice), les détecteurs à protons de recul permettant de plus de mesurer la distribution en énergie de la fluence, après étalonnage sur les références en énergie donnée par temps de vol. Ces détecteurs de référence sont utilisés avant et après chaque mesure ou série de mesures et des moniteurs, mesurant en permanence et de manière relative la fluence neutronique ou photonique, permettent de raccorder toute mesure à celle effectuée par les détecteurs de référence.

Par cette méthode, la fluence neutronique est déterminée avec une incertitude comprise entre 3 et $5 \%$.

\section{Conclusion}

L'IRSN offre un plateau technique très complet pour la production de tous les types de champ neutronique recommandé par les normes internationales pour l'étalonnage de tout instrument de détection des neutrons. L'ensemble de ces installations est ouvert à tout type d'utilisateur. 
À ce jour, seules les sources de l'irradiateur VAN GOGH sont intégrées dans la chaîne de métrologie française. L'objectif à moyen terme est de faire reconnaître l'ensemble des autres installations comme sources neutroniques de référence. Cela sera réalisé par l'organisation de comparaisons internationales sous l'égide du bureau international des poids et des mesures (BIPM) ou d'EUROMET (European Collaboration in Measurement Standards). Une telle comparaison a été réalisée en 2002 sur le dispositif CANEL/T400 et sera entreprise vers 2010 sur AMANDE après des exercices de comparaison plus modestes réalisés dans le cadre d'un contrat de collaboration avec les instituts de métrologie nationaux de Grande Bretagne (NPL) et d'Allemagne (PTB). Enfin, une comparaison internationale des références thermiques intégrant SIGMA se déroulera en 2006 et 2007.

Ce statut d'installations de référence sera également accompagné d'une généralisation des étalonnages accrédités COFRAC.

\section{RÉFÉRENCES}

Ambrosi P., Fantuzzi E., de Carvalho A.F., Delgado A., Lindborg L., Bartlett D.J. (2000) Procedures for routine individual dose assessment of external radiation within EU countries and Switzerland - status of harmonisation on 1 April 1999, Rad. Prot. Dosim. 89, 7-52.

Bordy J.M., Stadtmann H., Ambrosi P., Bartlett D.J., Christensen P., Hyvönen H. (2000) Performance test of dosimetric services in the EU member states and Switzerland for the routine assessment of individual doses (photon, beta, neutron), Rad. Prot. Dosim. 89, 107-154.

Briesmeister J.F. (Ed) (2000) MCNP ${ }^{\mathrm{TM}}$, LA-13709-M-version 4C.

CEI (1994) Norme CEI 1322, Radiation protection instrumentation, Installed dose equivalent rate meters, warning assemblies and monitors for neutron radiation of energy from thermal to $15 \mathrm{MeV}$.

CEI (2003) Norme CEI 61005, Instrumentation pour la radioprotection, Appareils de mesure de l'équivalent de dose ambiant neutron (ou de son débit d'équivalent de dose).

CEI (2005) Norme CEI 61526, Radiation protection instrumentation, Measurement of personal dose equivalents $\mathrm{Hp}(10)$ and $\mathrm{Hp}(0,07)$ for $\mathrm{X}$, gamma, neutron and beta radiations, Direct reading personal dose equivalent meters and monitors.

Chartier J.L., Posny F., Buxerolle M. (1992) Experimental assembly for the simulation of realistic neutron spectra, Rad. Prot. Dosim. 44, 125-130.

Gressier V., Guerre-Chaley J.-F., Lacoste V., Lebreton L., Pelcot G. (2002) Monitorage du champ neutronique de l'accélérateur SAMES T400 à l'aide de diodes silicium, Rapport IRSN SDOS/2002-011.

Gressier V., Pelcot G., Pochat J.L., Bolognese-Milstajn T. (2003) New IRSN facilities for neutron production, Nucl. Instrum. Meth. Phys. Res. A 505, 370-373.

Gressier V., Guerre-Chaley J.F., Lacoste V., Lebreton L., Pelcot G., Pochat J.L., Bolognese-Milstajn T., Champion D. (2004a) AMANDE: a new facility for monoenergetic neutron fields production between $2 \mathrm{keV}$ and $20 \mathrm{MeV}$, Rad. Prot. Dosim. 110, 49-52.

Gressier V., Lacoste V., Lebreton L., Muller H., Pelcot G., Bakali M., Fernández F., Tómas M., Roberts N.J., Thomas D.J., Reginatto M., Wiegel B., Wittstock J. (2004b) Characterisation of the IRSN CANEL/T400 facility producing realistic neutron fields for calibration and test purposes, $\mathrm{Rad}$. Prot. Dosim. 110, 523-527. 
ISO (1996) Norme ISO 4037-1, Rayonnements X et gamma de référence pour l'étalonnage des dosimètres et des débitmètres, et pour la détermination de leur réponse en fonction de l'énergie des photons, Partie 1 : Caractéristiques des rayonnements et méthodes de production.

ISO (2000) Norme ISO 12789-1, Rayonnements neutroniques de référence, Caractéristiques et méthodes de production de champs de neutrons simulant ceux de postes de travail.

ISO (2001) Norme ISO 8529-1, Rayonnements neutroniques de référence, Partie 1 : Caractéristiques et méthodes de production.

Lacoste V. (2005) Neutron spectrometry with Bonner spheres at workplaces in nuclear facilities at Ringhals (Sweden), EVIDOS Campaign n 3, Rapport IRSN SDE/05-02.

Lacoste V., Gressier V. (2004) Monte Carlo simulation of the IRSN CANEL/T400 realistic mixed neutron-photon radiation field, Rad. Prot. Dosim. 110, 123-127.

Lacoste V., Gressier V. (2005) Monte-Carlo simulation of the operational quantities at the realistic mixed neutron-photon radiation fields CANEL and SIGMA, European workshop on individual monitoring of ionising radiation, April 11-15, 2005, Vienna/Austria (à paraître).

Lacoste V., Muller H. (2004) Neutron Spectrometry with Bonner spheres at the VENUS Research reactor, EVIDOS Campaign no 2, Rapport IRSN SDE/04-056.

Lacoste V., Gressier V., Muller H., Lebreton L. (2004a) Characterisation of the IRSN graphite moderated Americium-Beryllium neutron field, Rad. Prot. Dosim. 110, 135-139.

Lacoste V., Reginatto M., Muller H. (2004b) Neutron Spectrometry with Bonner spheres at the Belgonucléaire MOX fuel processing plant, EVIDOS Campaign no 2, Rapport IRSN SDE/04034.

Marion J.B., Fowler J.L. (1960) Fast Neutron Physics. Interscience Publishers, London.

Pichenot G., Guldbakke S., Asselineau B., Gressier V., Itié C., Klein H., Knauf K., Lebreton L., Löb S., Pochon-Guérin L., Schlegel D., W. Sosaat (2002) Characterisation of spherical recoil proton proportional counters used for neutron spectrometry, Nucl. Instrum. Meth. Phys. Res. A 476 , 165-169.

Schmidt D., Asselineau B., Böttger R., Klein H., Lebreton L., Neumann S., Nolte R., Pichenot G. (2002) Characterization of liquid scintillation detectors, Instrum. Meth. Phys. Res. A 476, 186189.

Thomas D.J., Chartier J.L., Klein H., Naismith O.F., Posny F., Taylor G.C. (1997) Results of a Large Scale Neutron Spectrometry and Dosimetry Comparison Exercise at the Cadarache Moderator Assembly, Rad. Prot. Dosim. 70, 313-322.

Van Ryckeghem L., Lacoste V., Pelcot G., Pochat J.-L. (2002) Neutron calibration facilities of the IRSN research laboratory in external dosimetry, Proceeding of the 11 th International Symposium on Reactor Dosimetry, Brussels, Belgium, 18-23 August 2002, p. 528. 